\title{
Cryptogenic Cirrhosis, an Ambiguity of a Diagnosis: A Case Report of Progressive Cirrhosis of Undetermined Cause
}

\author{
Alvin Oliver Payus, MD, MRCP, ${ }^{1}$ Justin Leow Wen Hsiang, MD, MRCP, ${ }^{2}$ \\ Ong Hui Yun, $\mathrm{MD}^{3}$ and Neeraj Vinayakumar, MD, MRCP ${ }^{4}$ \\ ${ }^{1}$ Medicine Based Department, Faculty of Medicine and Health Science, Universiti Malaysia Sabah, Jalan UMS, 88400 Kota Kinabalu, Sabah, Malaysia \\ ${ }^{2}$ General Medicine Department, Oueen Elizabeth Hospital, 3 A Jalan Penampang, 88200 Kota Kinabalu, Sabah, Malaysia \\ ${ }^{3}$ General Medicine Department, Sultan Abdul Halim Hospital, 225 Bandar Amanjaya, 08000 Sungai Petani, Kedab, Malaysia \\ ${ }^{4}$ Department of Medicine, Sri Ramakrishna Ashrama Charitable Hospital, Thiruvananthapuram, 695011, Kerala, India
}

\begin{abstract}
Cirrhosis is the result of chronic liver disease due to a variety of causes. It is deemed to be cryptogenic when the leading cause cannot be identified despite extensive laboratory, radiological and pathological investigations. The prevalence of cryptogenic cirrhosis diagnosis has been dramatically reduced in recent years due to the advanced achievement in diagnostic medicine, whereby it is attributed to only about less than $5 \%$ of cirrhosis cases. Here, we present a case of a 16-year-old boy with nonsignificant family history, was not taking any regular medication, and presented with progressive intermittent jaundice for a few years due to liver cirrhosis. Although an extensive investigation has been done, the etiology of the cirrhotic liver was still unknown. He had no features to support nonalcoholic steatohepatitis. He was in Child's Grade B and prophylactically treated with a regular dose of propranolol to prevent portal hypertension complication while waiting for a liver transplant. This case report served the objective of showing that despite the advances in medical diagnostic techniques, cryptogenic cirrhosis is still used as a diagnosis in cases of chronic liver disease of unknown etiology.
\end{abstract}

Key Words: chronic liver disease, cryptogenic cirrhosis, nonalcoholic steatohepatitis

\section{INTRODUCTION}

Cirrhosis is a chronic, progressive, and incurable liver disease characterized by the formation of fibrosing alterations and abnormal hepatocellular nodules. ${ }^{1,2}$ It is a result of long-standing liver disease due to whatever causes. The determination of its etiology is essential as the specific treatment plan may vary depending on the cause. Some of the complications can be prevented if the progression of cirrhosis is altered. Among these complications, the most dreadful one is portal hypertension. When extensive laboratory, radiological, and pathological investigation has been done, and yet, the cause of the chronic liver disease is still unknown, the condition is called cryptogenic cirrhosis.

Cryptogenic cirrhosis is a term that is reluctantly given to any cirrhosis case when all other causes have been excluded. The term's use has been dramatically reduced from almost $50 \%$ to less than $5 \%$ in recent years. ${ }^{3}$ There has been

Corresponding author: Alvin Oliver Payus, MD, MRCP Medicine Based Department

Faculty of Medicine and Health Science

Universiti Malaysia Sabah

Jalan UMS, 88400 Kota Kinabalu, Sabah, Malaysia

Email:dralvinpayus@ums.edu.my a suggestion that cryptogenic cirrhosis and nonalcoholic steatohepatitis (NASH), which is a sequela of nonalcoholic fatty liver disease (NAFLD), is the same entity. Unfortunately, there is no definitive marker for NASH or NAFLD. Therefore, in the absence of obesity or history of diabetes, and 
Table 1. Laboratory investigation results showed raised transaminases and alkaline phosphatase level, and also direct hyperbilirubinemia, in keeping with an obstructive picture. On top of that, there was hypoalbuminemia with reversed albumin globulin ratio and prolonged prothrombin time as well, which show reduced productivity function of the liver. All the relevant infective and metabolic screening were negative.

\begin{tabular}{|c|c|c|}
\hline Investigation & Result & Normal Range \\
\hline Aspartate aminotransferase & $307 \mu / L$ & $5-35 \mu / L$ \\
\hline Alanine aminotransferase & $208 \mu / L$ & $5-35 \mu / L$ \\
\hline Alkaline Phosphatase & $418.1 \mu \mathrm{mol} / \mathrm{L}$ & $4-120 \mu / L$ \\
\hline Serum Bilirubin & $169.8 \mu \mathrm{mol} / \mathrm{L}$ & $0-13 \mu \mathrm{mol} / \mathrm{L}$ \\
\hline Direct bilirubin & $112.1 \mu \mathrm{mol} /$ & $0-8 \mu \mathrm{mol} / \mathrm{L}$ \\
\hline Albumin & $26 \mathrm{~g} / \mathrm{L}$ & $35-50 \mathrm{~g} / \mathrm{L}$ \\
\hline Globulin & $39 \mathrm{~g} / \mathrm{L}$ & $35-50 \mathrm{~g} / \mathrm{L}$ \\
\hline Prothrombin time & 22.2 seconds & $12.2-14.3$ seconds \\
\hline Alpha Fetoprotein & $5.1 \mathrm{U} / \mathrm{mL}$ & $0.74-7.38 \mathrm{U} / \mathrm{mL}$ \\
\hline Alpha-1 Antitrypsin & $1.83 \mathrm{~g} / \mathrm{L}$ & $0.90-2.0 \mathrm{~g} / \mathrm{L}$ \\
\hline Immunoglobulin A & $1.87 \mathrm{~g} / \mathrm{L}$ & $0.63-4.84 \mathrm{~g} / \mathrm{L}$ \\
\hline Immunoglobulin M & $13.45 \mathrm{~g} / \mathrm{L}$ & $5.40-18.22 \mathrm{~g} / \mathrm{L}$ \\
\hline Immunoglobulin G & $0.95 \mathrm{~g} / \mathrm{L}$ & $0.22-2.40 \mathrm{~g} / \mathrm{L}$ \\
\hline Serum Ceruloplasmin & $0.29 \mathrm{~g} / \mathrm{L}$ & $0.15-0.30 \mathrm{~g} / \mathrm{L}$ \\
\hline Urine Copper & $0.83 \mathrm{umol} / \mathrm{L}$ & $<0.90 \mathrm{umol} / \mathrm{L}$ \\
\hline Anti-Nuclear Antibody & \multicolumn{2}{|c|}{ Negative } \\
\hline Anti-Mitochondrial Antibody & \multicolumn{2}{|c|}{ Negative } \\
\hline Anti-Smooth Muscle Antibody & \multicolumn{2}{|c|}{ Negative } \\
\hline Anti-Liver Kidney Microsomal Antibody & \multicolumn{2}{|c|}{ Negative } \\
\hline HIV serology, HBsAg, Anti-HCV & \multicolumn{2}{|c|}{ Negative } \\
\hline Rapid Plasma Reagent & \multicolumn{2}{|c|}{ Negative } \\
\hline
\end{tabular}

any liver biopsy to support the diagnosis, to name NASH as the etiology often remains debatable as in this patient's case.

\section{CASE REPORT}

A 16-year old high school boy who was previously well, without any significant family history, presented with a long history of jaundice, which has been progressively worsening and associated with on and off body pruritus. Otherwise, he has no history of hematemesis, no high-grade fever, and no pale color stool or tea-colored urine. He denied any loss of appetite or weight; he denied taking traditional medication, health supplements, or any regular drugs, both prescribed and non-prescribed.

On examination, he has a lean body habitus and was deeply jaundiced, but otherwise not pale, no palpable lymph nodes, and no pedal edema. Numerous spider nevi and multiple scratch marks were noted. His abdomen was soft and not tender. There was no palpable mass nor organomegaly; no ascites and bowel sound was present. The examination of other systems was unremarkable.

A series of laboratory investigations were done (as shown in Table 1). He has a raised transaminases and alkaline phosphatase levels, and also direct hyperbilirubinemia which in keeping with cholestatic hepatitis picture. On top of that, there was hypoalbuminemia with reversed albumin globulin ratio, and prolonged prothrombin time as well, which showed reduced productivity function of the liver. A battery of infective and metabolic screening for chronic liver disease was done, but all were negative.

Ultrasound of the hepatobiliary system (as shown in Figure 1 and Figure 2) showed the liver was nodular and had an irregular outline with coarse echotexture - multiple round hypoechoic nodules of various sizes scattered throughout the liver parenchyma. The biliary tree was not dilated. The gallbladder has an irregular outline, but no thickened wall, no mass, and no stones were noted. The portal vein was patent and not dilated. The spleen was homogenously enlarged and measuring $14 \mathrm{~cm}$ with no focal lesion. The pancreas and both kidneys were normal. No ascites and the para-aortic nodes were not enlarged.

A multiphase CT scan of the liver showed an irregular liver margin measuring $12.8 \mathrm{~cm}$. No focal enhancing lesion in arterial and venous phase. No hypodense liver lesions. Portal vein measured $1.2 \mathrm{~cm}$ and is well opacified. The porta hepatic lymph node measured $1.3 \mathrm{~cm}$. The gallbladder was well distended. The spleen was enlarged, measuring $15.1 \mathrm{~cm}$ with splenic varices. Pancreas, adrenals, and kidneys 

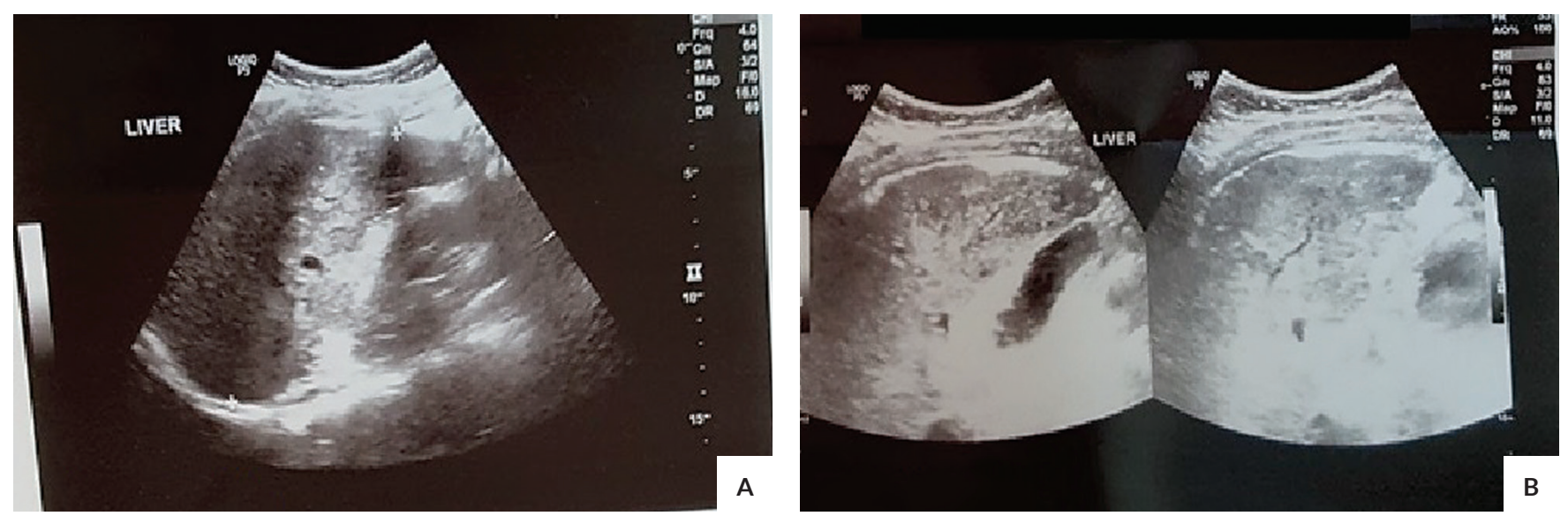

Figure 1. Ultrasound of the Hepatobiliary system showed irregular and nodular liver outlines with coarse echotexture. Multiple round hypoechoic nodules of various sizes scattered throughout the liver parenchyma. The biliary tree was not dilated, and gallbladder has an irregular outline, but otherwise has no thickened wall, no mass, and no stones noted.

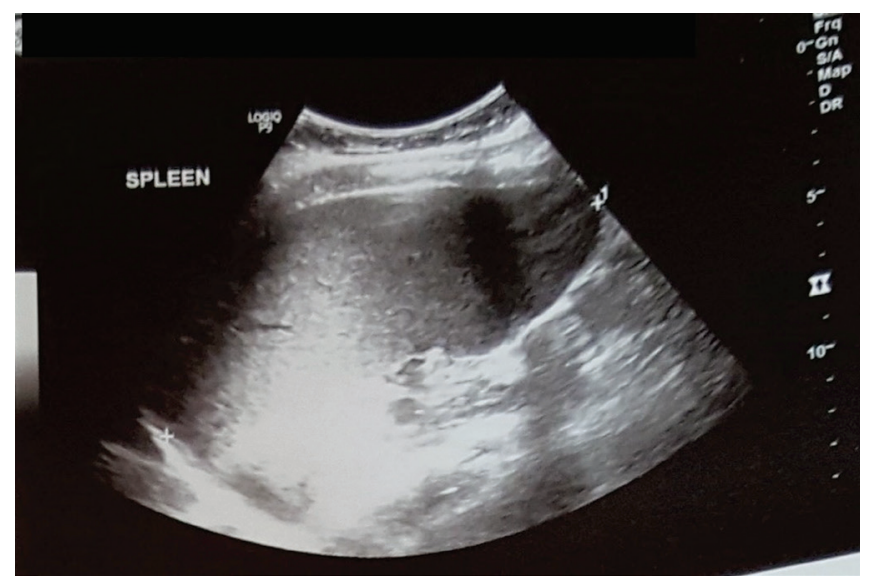

Figure 2. Ultrasound of the Hepatobiliary system (HBS) showed spleen was homogenously enlarged and measuring about $14 \mathrm{~cm}$ with no focal lesion.

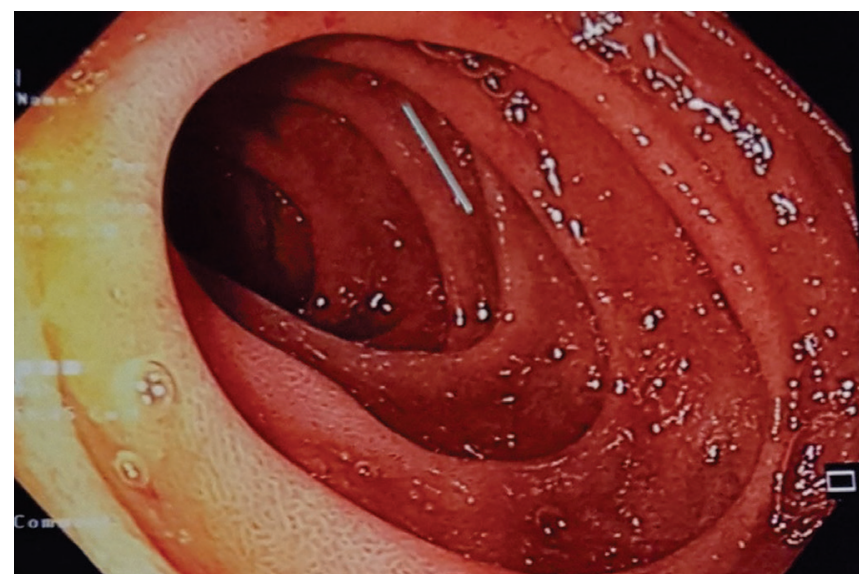

Figure 3. Esophagogastroduodenoscopy showed a normal second part of the duodenum. were normal. Esophagogastroduodenoscopy (as shown in Figures 3 and 4) showed grade I esophageal varices and a mild portal hypertensive gastropathy over the body and antrum. The fundus and duodenum were normal.

The patient is currently in Child's grade B liver cirrhosis. He was started on regular dose of propranolol $40 \mathrm{mg}$ twice daily to reduce the risk of portal hypertension complication. Liver biopsy was done and reported as fibrotic micronodular changes in the absence of steatosis, cholestasis, nor any stains for copper, iron, and alpha- 1 antitrypsin globules. He was listed for a liver transplant as we believe he is the right candidate given his young age and little complication from the condition. However, without knowing the exact cause of his illness, it is impossible to predict his condition's longterm outcome after the liver transplant, especially in terms of recurrent rate. Therefore, further investigations and studies are warranted even after the liver transplant to identify the leading cause for the reasons mentioned above.

\section{DISCUSSION}

Cirrhosis is a result of chronic liver disease due to many causes. It can be defined as a diffuse, long-standing, and progressive liver disease, characterized by the distortion of liver micro-architecture and the formation of fibrosis and abnormal regenerative nodules in the liver. ${ }^{1}$ Common causes of cirrhosis include chronic viral hepatitis, alcoholic liver disease, nonalcoholic fatty liver disease (NAFLD), and hemochromatosis. ${ }^{2}$ In some cases, when the leading cause of cirrhosis remains unknown despite extensive clinical, serological, radiological, and pathological evaluations; it is called cryptogenic cirrhosis. It is a diagnosis of exclusion given only when there is no other known etiology can be identified. Previously, the diagnosis was widely used, which was $30 \%$ to $50 \%$. Due to the advances in the diagnostic medicine and development of multiple new serology tests, the prevalence 

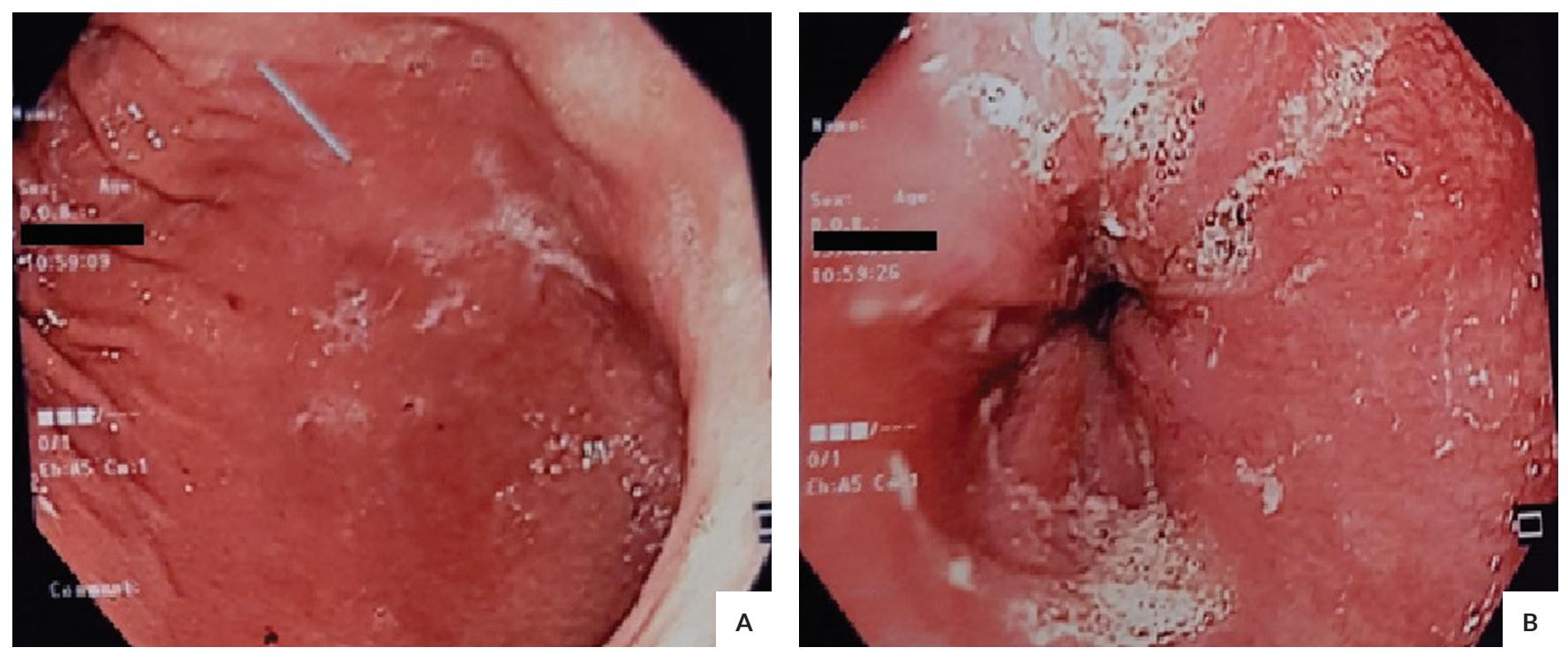

Figure 4. Esophagogastroduodenoscopy grade I esophageal varices and mild portal hypertensive gastropathy with a mosaic-like and reticular cobblestone pattern.

of diagnosing cryptogenic cirrhosis has been dramatically reduced to an estimated less than $5 \% .^{3}$

In recent years, there has been a suggestion that cryptogenic cirrhosis and nonalcoholic steatohepatitis (NASH), sequelae of NAFLD are the same entity. ${ }^{4}$ This suggestion arose because most patients diagnosed with cryptogenic cirrhosis showed a higher obesity incidence and diabetes mellitus type 2 compared to patients with cirrhosis of other causes. ${ }^{5}$ Therefore, there is a trend among some physicians to use the diagnosis of cryptogenic cirrhosis and $\mathrm{NASH}$ interchangeably. When NASH emerged as one of the leading causes of chronic liver disease in the western world, about two decades ago, there have been suggestions that patients with cryptogenic cirrhosis may be an evolved case from NASH, especially among patients with a risk factor such as obesity, diabetes mellitus, and others. ${ }^{6}$ However, the present study showed that despite the increased awareness of NASH as an important cause of cirrhosis, many patients are currently being listed for liver transplantation with a diagnosis of cryptogenic cirrhosis. ${ }^{7}$ This happens because these patients did not fulfill the criteria to be diagnosed as NASH or NAFLD. There is no definitive diagnostic marker to diagnose NASH, whereby it is diagnosed primarily based on suggestive history on top of the pathological test. Therefore, it was more than likely that those patients currently listed as having cryptogenic cirrhosis, are due to actual unknown cause of chronic liver disease, rather than an evolved complication from NASH. ${ }^{8}$

As with cirrhosis due to other causes, cryptogenic cirrhosis was also associated with complications such as portal hypertension, portal vein thrombosis, spontaneous bacterial peritonitis, and development of hepatocellular carcinoma (HCC). According to Stine JG et al., who studied the difference in the prevalence of complications between NASH-related cirrhosis and cryptogenic cirrhosis, the prevalence of HCC and portal vein thrombosis were higher in cirrhosis associated with NASH as compared to cryptogenic cirrhosis. In contrast, for spontaneous bacterial peritonitis and portal hypertension-related complications (such as ascites, encephalopathy, hepatorenal syndrome, and others), the prevalence was similar in both conditions. ${ }^{9}$

Treatment for cryptogenic cirrhosis can only be done in comprehensive measures since the exact etiology is unknown. These measures include hindering the progression, stabilizing liver function to prevent decompensation, managing portal hypertension and its complication, and ultimately liver transplantation. As the rate of progress and recurrence of the condition highly depends on the causes, one can only suggest avoiding common risk factors such as alcohol abuse and medication that potentially have an adverse effect on the liver.

This report presented a patient who came in with progressive jaundice with intermittent obstructive symptoms secondary to chronic liver disease. He was already in Child's $\mathrm{B}$ upon the first presentation. A long list of laboratories, radiological, and pathological investigations have been done to identify the etiology, but everything came back negative. The patient has no associated features that can fit him into NASH, such as obesity, diabetes mellitus, high cholesterol, and hypertension. He was treated with a regular dose of non-selective beta blockers to control his portal hypertension and was under the waiting list for liver transplantation. This case report's objective was to support the recent study, which showed cryptogenic cirrhosis should not be considered the same entity as NASH, especially in a case where the patient has no risk factor that can fit into the criteria of NASH. Further studies are needed to establish the unknown causes of cirrhosis. 


\section{CONCLUSION}

In conclusion, many chronic liver diseases can lead to cirrhosis. Moreover, it is essential to determine the etiology because the overall management and long-term outcome of liver cirrhosis are highly dependent on the leading causes. However, despite the advanced achievement in the medical field, we still failed to identify the etiology of some liver cirrhosis cases. Hence, cryptogenic cirrhosis will have to be ambiguously used as a working diagnosis. Furthermore, as suggested by a recent study, cryptogenic cirrhosis should not be considered the same as cirrhosis related to NASH. Therefore, further investigations are required to identify unknown causes of cirrhosis.

\section{Ethical Consideration}

Written informed consent was obtained from the patient to publish this case report.

\section{Acknowledgment}

The authors would like to thank the patient and families for their cooperation with regard to this report. The author would also like to thank the Director-General of Health Malaysia for his permission to publish this article.

\section{Statement of Authorship}

All authors participated in data collection and analysis, and approved the final version submitted.

\section{Author Disclosure}

All authors declared no conflicts of interest.

\section{Funding Source}

No funding support.

\section{REFERENCES}

1. Anthony PP, Ishak KG, Nayak NC, Poulsen HE, Scheuer PJ, Sobin LH. The morphology of cirrhosis. Recommendations on definition, nomenclature, and classification by a working group sponsored by the World Health Organization. J Clin Pathol 1978 May; 31(5):395-414. DOI: $10.1136 /$ jcp.31.5.395

2. Heidelbaugh JJ, Bruderly M. Cirrhosis and chronic liver failure: part I. Diagnosis and evaluation. Am Fam Physician 2006 Sep 1; 74(5): 756-762.

3. Charlton MR, Kondo M, Roberts SK et al. Liver transplantation for cryptogenic cirrhosis. Liver Transpl Surg. 1997 Jul;3(4):359-364. DOI: $10.1002 / 1 t .500030402$.

4. Clark JM, Diehl AM. Nonalcoholic fatty liver disease: an underrecognized cause of cryptogenic cirrhosis. JAMA. 2003 Jun 11;289(22):3000-3004. DOI: 10.1001/jama.289.22.3000

5. Poonawala A, Nair SP, Thuluvath PJ. Prevalence of obesity and diabetes in patients with cryptogenic cirrhosis: A case-control study. Hepatology. 2000 Oct;32(4 Pt 1):689-692. DOI: 10.1053/jhep.2000.17894.

6. Younossi ZM, Otgonsuren M, Henry L, Venkatesan C, Mishra A, Erario M, Hunt S. Association of nonalcoholic fatty liver disease (NAFLD) with hepatocellular carcinoma (HCC) in the United States from 2004 to 2009. Hepatology. 2015 Dec;62(6):1723-1730. DOI: 10.1002/hep.28123.

7. Wong RJ, Aguilar M, Cheung R, Perumpail RB, Harrison SA, Younossi $\mathrm{ZM}$, Ahmed A. Nonalcoholic steatohepatitis is the second leading etiology of liver disease among adults awaiting liver transplantation in the United States. Gastroenterology. 2015 Mar;148(3):547-555. DOI: $10.1053 /$ j.gastro.2014.11.039.

8. Thuluvath PJ, Kantsevoy S, Thuluvath AJ, Savva Y. Is cryptogenic cirrhosis different from NASH cirrhosis? J Hepatol. 2018 Mar;68(3):519-525. DOI: 10.1016/j.jhep.2017.11.018.

9. Stine JG, Shah NL, Argo CK, Pelletier SJ, Caldwell SH, Northup PG. Increased risk of portal vein thrombosis in patients with cirrhosis due to nonalcoholic steatohepatitis. Liver Transpl. 2015 Aug;21(8):1016-1021. DOI: 10.1002/1t.24134.

\section{The Acta Medica Philippina is now accepting limited advertising for its front and back cover (colored), as well as for available spaces in some of its pages, as appropriate. For inquiries and submission of proposals, please email us at actamedicaphilippina.upm@up.edu.ph}

